\title{
Patient experience of uncertainty in cancer genomics: a systematic review
}

\author{
Nicci Bartley, McEval ${ }^{1}{ }^{1}$, Christine Napier, $\mathrm{PhD}^{2}$, Megan Best, $\mathrm{PhD}^{1}$ and Phyllis Butow, $\mathrm{PhD}^{1}$
}

While genomics provides new clinical opportunities, its complexity generates uncertainties. This systematic review aimed to summarize what is currently known about the experience of uncertainty for adult patients undergoing cancer genomic testing. A search of five databases (2001 to 2018) yielded 6508 records. After removing duplicates, abstract/title screening, and assessment of full articles, ten studies were included for quality appraisal and data extraction. Qualitative studies were subjected to thematic analysis, and quantitative data were summarized using descriptive statistics. Cancer genomic results reduced uncertainty for patients regarding treatment decisions but did not reduce uncertainty in the risk context. Qualitative and quantitative data synthesis revealed four themes: (1) coexisting uncertainties, (2) factors influencing uncertainty, (3) outcomes of uncertainty, and (4) coping with uncertainty. Uncertainty can motivate, or be a barrier to, pursuing cancer genomic testing. Appraisal of uncertainty influences the patient experience of uncertainty, the outcome of uncertainty for patients, as well as the coping strategies utilized. While this systematic review found that appraisal of uncertainty is important to the patients' experience of uncertainty in the cancer genomic context, more mixed methods longitudinal research is needed to address the complexities that contribute to patient uncertainty across the process.

Genetics in Medicine (2020) 22:1450-1460; https://doi.org/10.1038/s41436020-0829-y

Keywords: uncertainty; cancer; genomic; patient perspective

\section{INTRODUCTION}

There were an estimated 18.1 million new cases of cancer in 2018. ${ }^{1}$ Cancer is caused by changes in genes either inherited from parents (germline), or developed over a person's lifetime due to errors in cell division or exposures in their environment (somatic). ${ }^{2}$ Cancer genetic testing focuses on single genes with a known function, for example BRCA1 and $B R C A 2$ gene testing to identify risk of hereditary breast and ovarian cancer syndrome testing. Genomic testing refers to a broader investigation of genetic material with no specific gene target, for example multigene panel, genome sequencing, or exome sequencing, which include genes that are not fully understood in terms of cancer risk or management strategies. ${ }^{3}$ Genomic testing provides hope for improvement in cancer prevention, by helping to identify individuals at increased risk of a broad range of cancers; ${ }^{4}$ and reducing mortality and morbidity, through the identification of specific pathogenic variants to guide treatment. ${ }^{5}$ Genomic testing also introduces challenges, ${ }^{2}$ for example the uncertainty that is inherent in this new technology. ${ }^{6}$

Illness uncertainty, a patients' inability to determine the meaning of illness-related events, ${ }^{7}$ can be a result of ambiguity (conflicting, incomplete, or inadequate information); complexity (difficult to understand information); and unpredictability (likelihood or risk of future outcome of the disease). ${ }^{7-9}$
Uncertainty in illness has been associated with anxiety, depression, and distress. ${ }^{7,10,11}$

The complexity of genomic testing introduces new scientific, practical, and personal uncertainties specific to this process. ${ }^{6}$ Genomic testing can reveal variants that are (1) relevant to the target cancer versus secondary findings (relevant to other cancers or diseases), (2) clinically actionable versus non-clinically actionable (no proven treatments), and (3) of unknown or uncertain significance., ${ }^{2,12}$ Treatments targeting actionable genomic results are often experimental. Thus, while reducing uncertainty can be a motivator for pursuing genomic testing, ${ }^{13}$ results have the potential to increase uncertainty.

Existing uncertainty research has focused on patient uncertainty when undergoing single-gene testing (such as $B R C A 1 / 2$ testing for specific hereditary cancer syndromes), ${ }^{14-16}$ the uncertainty related to the science of genomics, or uncertainty experienced by health-care professionals in relation to interpreting results, and communicating uncertain information. ${ }^{17}$ Little is known about the patient experience of uncertainty when undergoing cancer genomic testing, and the impact of this uncertainty on patient psychosocial outcomes. Furthermore, there has been no synthesis of the extant literature to aid identification of gaps and guide future research.

\footnotetext{
${ }^{1}$ School of Psychology, The University of Sydney, Sydney, Australia; ${ }^{2}$ Cancer Theme, Garvan Institute of Medical Research, Darlinghurst, Australia. Correspondence:
} Nicci Bartley (nicole.bartley@sydney.edu.au)

Submitted 11 December 2019; revised 21 April 2020; accepted: 27 April 2020

Published online: 19 May 2020 
To meet this need, we conducted a systematic literature review aimed at identifying and synthesizing what is currently known about the patient experience of uncertainty when undergoing cancer genomic testing. Our review questions were:

1. What type of uncertainty do patients experience?

2. To what extent do patients experience uncertainty?

3. What are the factors that influence uncertainty?

4. What is the impact of uncertainty for these patients?

\section{MATERIALS AND METHODS}

\section{Study design}

This systematic review protocol was designed using the Preferred Reporting Items for Systematic Reviews (PRISMA) standards ${ }^{18}$ and ENTREQ guidelines. ${ }^{19}$ The review was registered with PROSPERO, registration CRD 42018084301.

\section{Search strategy}

A literature search of peer-reviewed publications was conducted using five databases (Scopus, Ovid MEDLINE, Embase, PsycINFO, and CINAHL). Search terms were a combination of keywords and $\mathrm{MeSH}$ terms relating to uncertainty, cancer, and genomics (Supplementary material). Qualitative, quantitative, and mixed methods studies (including hypothetical studies), published in English from January 2001 to June 2018, were included. The year 2001 was set as the outside date boundary because it was the year the initial sequencing and analysis of the human genome was completed, ${ }^{20}$ marking an important milestone in the field of genetics.

Articles were excluded if they assessed uncertainty in the context of single-gene testing, for example BRCA1 or BRCA2 testing, or in relation to illnesses other than cancer; or focused on health-care professionals, or pediatric or adolescent populations. Unpublished reports, conference abstracts, systematic reviews, and dissertations were excluded. Reference lists of accepted articles were hand-searched for additional relevant publications.

\section{Data extraction and assessment of study quality}

Data extracted included study design, study objective, measures used, cancer genomic test, sample size and characteristics, and key findings. Data were extracted independently by two authors (N.B. and C.N.). A 30\% overlap was extracted by a third author (P.B.) to ensure reliability. All studies included were evaluated using the Joanna Briggs Institute Critical Appraisal Tools, ${ }^{21}$ for risk of bias across the study design, conduct, and analysis. One author (N.B.) independently assessed study quality with a $30 \%$ overlap assessed for quality by a second author (P.B.). Any disparities were resolved through discussion. No studies were excluded based on quality.

\section{Analysis}

Analysis was conducted using a three-stage synthesis process that combined qualitative and quantitative studies comprehensively and rigorously to answer the review questions. ${ }^{22}$ As meta-analysis was not possible due to the heterogeneity of study outcomes, the first analysis focused on identifying and summarizing the quantitative data. The second analysis involved thematic synthesis ${ }^{23}$ of the qualitative studies. Line-by-line coding of the results sections of an initial two qualitative studies was conducted (N.B., M.B., and P.B.) to develop a coding tree and themes. This coding structure was then applied to the remaining qualitative studies (N.B.) with new codes created and discussed by the authors. Finally, a matrix was used to compare the qualitative and quantitative findings from the first two stages to answer the review research questions.

\section{RESULTS}

The search strategy identified 6508 articles which were exported to EPPI-Reviewer 4. After removing duplicates, the remaining 5154 records were screened by title and abstract by two authors (N.B. and C.N.) with a $10 \%$ overlap. The two authors had an overall agreement rate of $98 \%$ (fixed marginal kappa of 0.97; free marginal kappa of 0.98). The full texts of 111 retained papers were retrieved for screening by three authors (N.B., C.N., and M.B.), with 10\% double screened by the fourth author (PB) (Fig. 1). Of these, nine studies met eligibility criteria. One additional study was identified through a hand search of the eligible article reference lists, and ten studies were included for data extraction and quality assessment.

\section{Article characteristics}

Table 1 summarizes the ten included studies and their key findings regarding patient uncertainty. Only two studies assessed uncertainty as a primary outcome. ${ }^{24,25}$ Two studies assessed hypothetical intention to undergo cancer genomic testing: ${ }^{24,26}$ the remainder focused on participants' experience while pursuing cancer genomic testing. ${ }^{25,27-33}$ The majority of studies were quantitative $(n=5)$, cross-sectional $(n=6)$, and conducted in English-speaking Western countries $(n=8)$.

There was a scarcity of literature investigating the patient experience of uncertainty in cancer genomics, with only one or two studies published for each type of genomic test. As such we analyzed uncertainty across the range of genomic tests: genetic test for bowel/breast cancer susceptibility (hypothetical), ${ }^{24}$ DNA microarray to direct treatment, ${ }^{32}$ Oncotype DX 21-gene assay to predict chemotherapy benefit, ${ }^{27,29}$ single-nucleotide polymorphism (SNP) testing for colorectal cancer susceptibility (hypothetical), ${ }^{26}$ exome sequencing, ${ }^{33}$ 25-gene test for cancer susceptibility, ${ }^{28,31}$ multigene panel tests for cancer susceptibility, ${ }^{30}$ and mismatch repair gene test for cancer susceptibility. ${ }^{25}$

\section{Sample characteristics}

There were a total of 1906 participants across all included studies, with the number of participants across the studies ranging from 19 to 1000. Participants' ages across the ten studies ranged from 18-90 years. All studies comprised a 


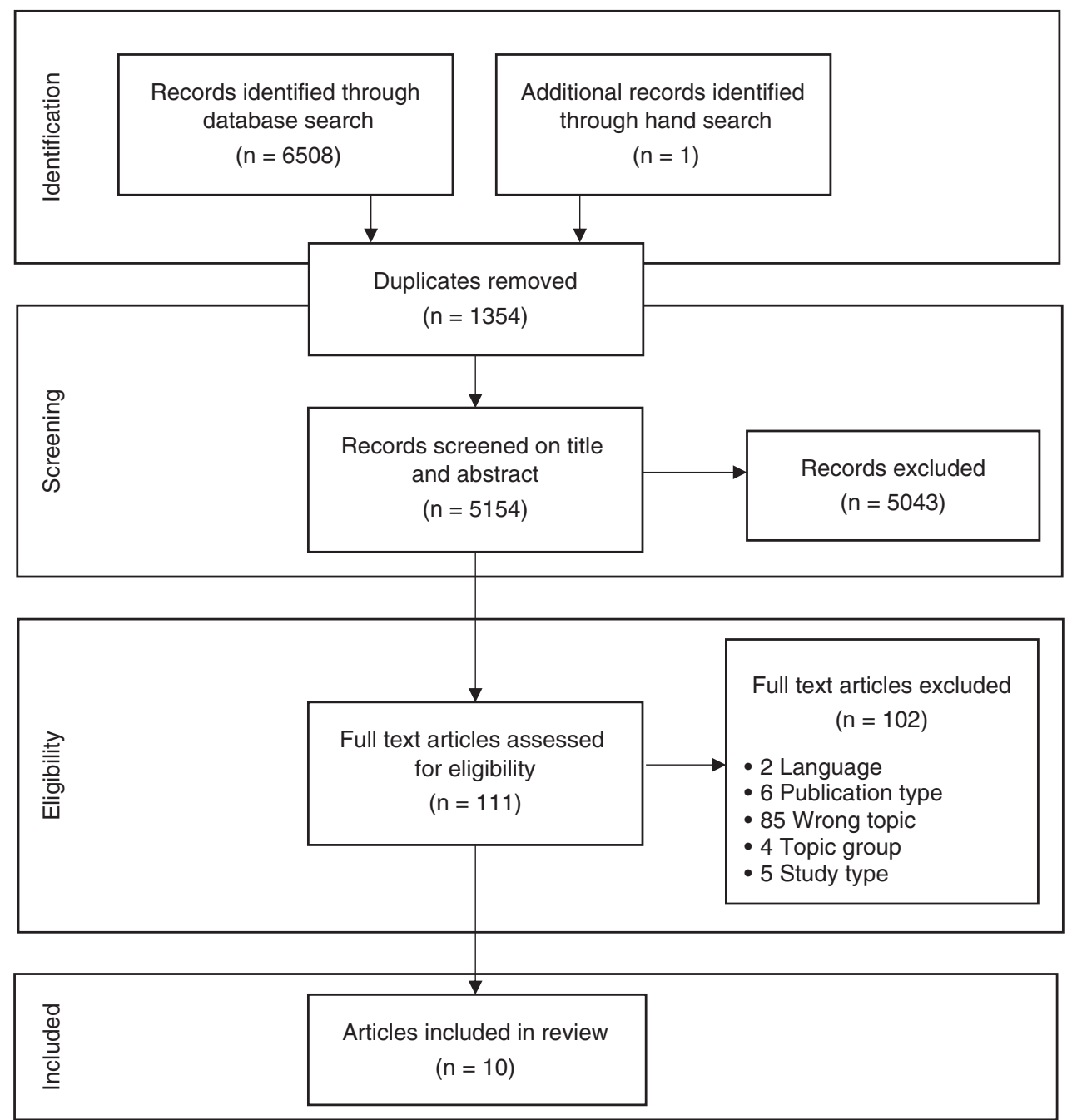

Fig. 1 Preferred Reporting Items for Systematic Reviews (PRISMA) flow diagram of the literature screening.

majority female participant sample, and the seven studies that reported participants' cancer diagnosis mainly included participants with a personal or family history of breast and/or ovarian cancer, and to a lesser extent colorectal cancer.

\section{Uncertainty measures}

Three separate measures of uncertainty were used. The attitude toward uncertainty scale ${ }^{24}$ measures attitude toward uncertainty in the medical testing context, where higher scores (mean of seven items; possible range 1-5) reflect a negative attitude toward uncertainty. The Multidimensional Impact of Cancer Risk Assessment (MICRA) uncertainty subscale $^{34}$ measures uncertainties associated with testing results and future plans, where higher scores (sum of nine items; possible range $0-45$ ) indicate greater uncertainty. The Decisional Conflict Scale ${ }^{35}$ measures uncertainties about a course of action, factors contributing to uncertainty, and perceptions of effective decision making, where higher scores (mean of ten items multiplied by 25 ; possible range $0-100)^{36}$ are associated with higher levels of uncertainty.

\section{Extent of uncertainty}

The quantitative synthesis showed that mean uncertainty scores were midrange for participants when deciding to undergo genomic testing for cancer risk information ${ }^{28}$ and did not change post-result disclosure. ${ }^{28,31}$ In the cancer treatment context, genomic results reduced participant treatment decisional uncertainty, i.e., to have (or not have) chemotherapy. $^{27,29}$

\section{Qualitative and quantitative synthesis}

Synthesis of quantitative and qualitative results revealed four themes (Table 2): (1) coexisting uncertainties, (2) factors influencing uncertainty, (3) outcomes of uncertainty, and (4) coping with uncertainty.

\section{Coexisting uncertainties}

Multiple interrelated uncertainties can exist for patients considering or undergoing cancer genomic testing. Uncertainty can be a motivation for ${ }^{26,33}$ or barrier to ${ }^{24,28}$ pursuing cancer genomic testing. When hypothetically considering genomic testing for cancer risk, $15.75 \%$ of participants of one 

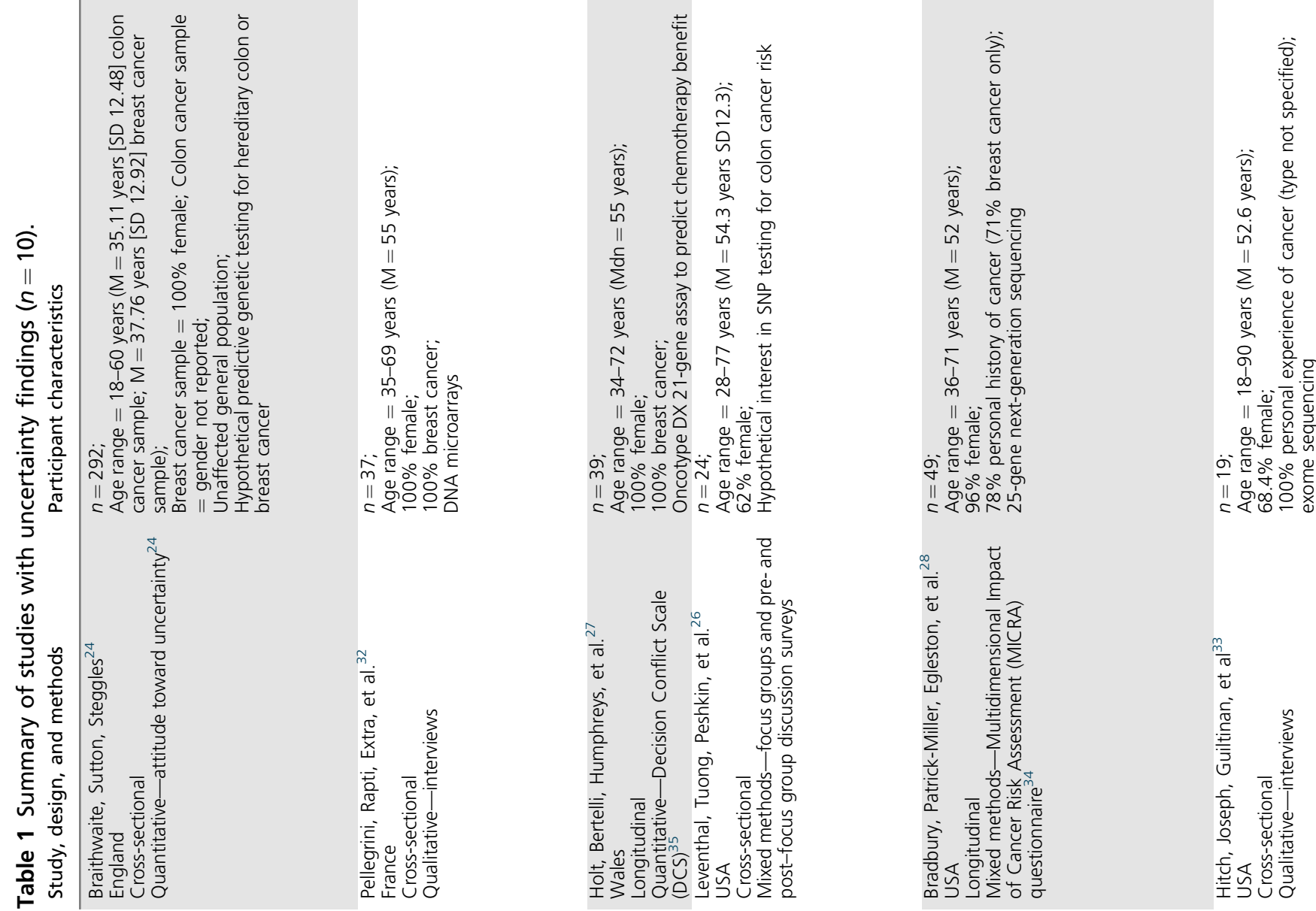


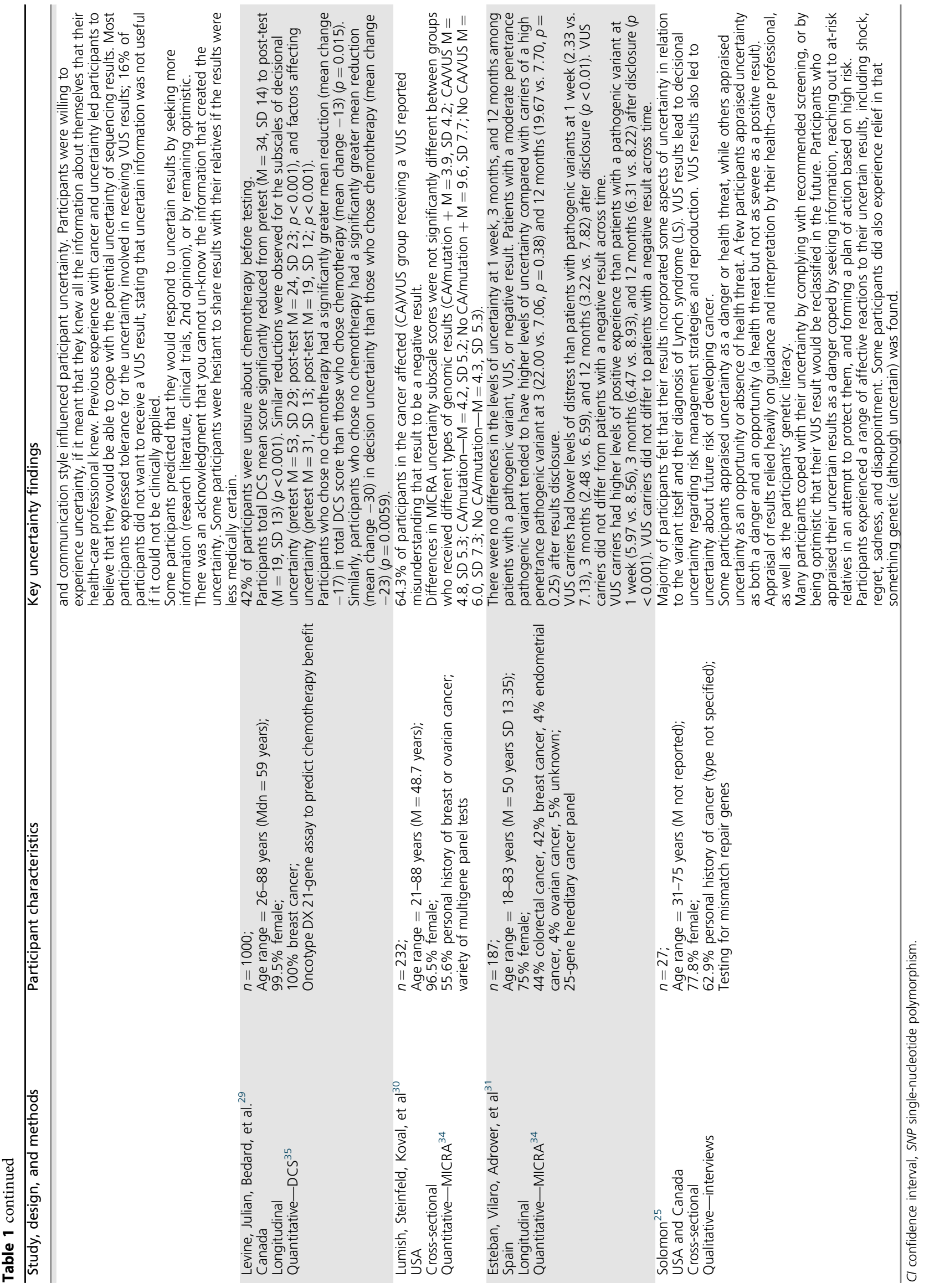


Table 2 Quantitative and qualitative analysis matrix.

Review question

What types of uncertainty do patients experience when undergoing cancer genomic testing?

To what extent do patients experience uncertainty when undergoing cancer genomic testing?

What are the factors that influence uncertainty in patients undergoing cancer genomic testing?
Quantitative synthesis

Participants experienced uncertainty about undergoing genomic testing, ${ }^{24}$ concerned about potential uncertainty or unclear utility of results. ${ }^{28}$ Participants with more negative attitudes toward uncertainty were more likely to choose to participate in hypothetical genomic testing for colon or breast cancer risk. ${ }^{24}$
Qualitative synthesis

Reducing uncertainty about personal and relatives' cancer risk was a motivation for undergoing genomic testing. 26,33

When considering genomic testing participants experience multiple uncertainties (future risk, ${ }^{25,26}$ clinical utility ${ }_{5}^{26,33}$ ambiguous results, ${ }^{25}$ decisional uncertainty, ${ }^{25}$ reactions to results, ${ }^{26,33}$ and collateral damage ${ }^{26,28}$ ).

Uncertainties are interrelated and conflicting; reducing one uncertainty can create a different uncertainty. ${ }^{28}$

Some participants were willing to risk uncertainty for any risk information, while others felt it was not worth the additional worry. ${ }^{26}$

Participants undergoing genomic testing for risk information had midrange baseline mean uncertainty scores, $^{28}$ and uncertainty did not change over time. 28,31

Genomic results reduced treatment decision uncertainty. 27,29

Attitude toward uncertainty was correlated with behavioral intention, subjective norm, and attitude toward testing. ${ }^{24}$

A less informed choice about genomic testing was associated with greater uncertainty. ${ }^{28}$

Previous experience with genetic testing did not influence participants' mean baseline uncertainty. ${ }^{28}$ Genomic result (positive, negative, VUS) did not influence uncertainty in the risk context. Moderate penetrance pathogenic variant carriers tended to have more uncertainty compared with high penetrance pathogenic variant carriers 12 months postresults. $^{3}$

- Health-care professionals' credentials
What are the outcomes of uncertainty in patients undergoing cancer genomic testing?
Participants with uncertain genomic results had lower levels of distress and higher levels of positive experience than participants with pathogenic variants up to 12 months' post-result disclosure. VUS carriers did not differ from participants with a negative result for both distress and positive experience following result disclosure. ${ }^{31}$
Factors affecting appraisal and experience of uncertainty include:

communication approach and style $25,26,28,32,33$

- Genetic literacy (misconceptions; inadequate

information). $25,26,28,32,33$

- Previous experience with uncertainty, disease, and perceived self-efficacy in coping with

uncertainty. ${ }^{2}$

- Willingness to experience uncertainty to know all the information about themselves that their healthcare professional know. ${ }^{33}$

- Types of results can increase or decrease uncertainty. Some participants expressed a tolerance for the uncertainty involved in receiving VUS results; ${ }^{33}$ however, VUS can also increase uncertainty about the future.

- A trust in science influenced treatment decisions certainty. $^{32}$

Experience of uncertainty depends on the appraisal of uncertainty. ${ }^{25,26}$ Some participants appraise uncertainty as a danger or health threat, while others appraise uncertainty as an opportunity or absence of health threat. A few participants appraise uncertainty as both a danger and an opportunity (a health threat but not as severe as a positive result). ${ }^{25}$

Uncertainty is ongoing, cannot un-know information that created uncertainty.

Sharing uncertain results with relatives would be difficult and hesitant to do so. ${ }^{33}$

Participants who received uncertain results experienced a range affective reactions to their results, including shock, regret, sadness, and disappointment. ${ }^{25,26,32}$ Some participants did also experience relief in that something genetic (although uncertain) was found. ${ }^{25}$

uncertain) was found. uncertain results by seeking more information, or by remaining optimistic. ${ }^{33}$

Participants coped with their uncertainty by complying with recommended screening, or by being optimistic that their VUS result would be reclassified in the future. Where participants appraised their uncertain results as a danger, they coped by seeking information, reaching out to at-risk relatives in an attempt to protect them, and forming a plan of action based on high risk. ${ }^{25}$

VUS variant of uncertain significance, $M$ mean, $M d n$ median.

study were unsure if they would pursue genomic testing for breast and colon cancer risk. ${ }^{24}$ These participants reported on average a somewhat negative attitude toward uncertainty $(M=2.63, S D 14.41)$; those with a more negative attitude toward uncertainty were more likely to choose to pursue testing, ${ }^{24}$ motivated by a desire to reduce uncertainty related to their own and their relatives' future risk of cancer. ${ }^{26,33}$

"I'd like to know if that's where I'm headed. Maybe there's nothing I could do about it-that doesn't matter. I think 
there's a benefit to knowing as much as you can about yourself." 26

Undergoing (or considering) genomic testing can create uncertainties about future risk, ${ }^{25,26}$ clinical utility, ${ }^{26,28,33}$ collateral damage, ${ }^{26,28}$ potential emotional and behavioral reactions to results, ${ }^{26,33}$ as well as decisional uncertainty. ${ }^{25}$ Ambiguity surrounding the meaning of a variant of uncertain significance (VUS) result can create uncertainty about future risk for participants, ${ }^{25}$ due to conflicting views and interpretation, and lack of conclusive information about the variant. Thus, some participants were motivated against undergoing genomic testing by high perceived uncertainty regarding clinical utility of genomic results, particularly if the results indicated a small increased cancer risk or were of uncertain significance. ${ }^{26,33}$

"So it's just-it is an ambiguity, and it makes me wonder, you know like I said, do I really have it or not?"25

"Unless you might have something that will impact your health in the future, I don't know if it's good or useful for just anybody to get to see their whole genetic makeup." 33

Participants who received VUS results experienced further uncertainty related to decision making around reproduction and preventive surgery. ${ }^{25}$

Participants expressed uncertainty about potential collateral damage or negative implications of undergoing genomic testing, such as impact on insurance, employment, and privacy. ${ }^{26,28}$ Participants also acknowledged that they were uncertain about how they would react to their genomic results, stating that results could have a negative impact on their emotions and also behavior. ${ }^{26,33}$

"I guess I'm concerned that in some ways I might change the way that I'm living my life now unintentionally. Not even consciously, but having that knowledge may potentially change what I'm doing now that I don't actually want to change...."33

The above uncertainties are interrelated and can be in conflict. Acknowledging that the science is still evolving, participants reported feeling both certainty and uncertainty from their genomic results, and that decreasing one uncertainty can increase another type of uncertainty. ${ }^{28,32}$

“There's a black cloud over my head if I don't get the testing because it's like you're constantly wondering what is causing it, but it's a little black cloud if I get it the testing and they find something because then I know it's genetic and there's nothing I can do...." ${ }^{28}$

Some participants were willing to risk uncertainty for any risk information, while others felt it was not worth the additional worry. ${ }^{26}$

\section{Factors influencing uncertainty}

A number of factors influenced participants' experience of uncertainty related to genomic testing. Information seekers showed higher tolerance for uncertainty, preferring to know as much as possible about their risks even if that information is uncertain. ${ }^{26,33,37}$ Some participants also had a preference for knowing their genomic information (even uncertain results) over paternalistic secrecy, stating that if medical professionals know genomic results, that information should be shared with the individual. ${ }^{33}$

Participants' own genomic literacy ${ }^{25,26,28,32,33}$ influenced their experience of uncertainty, with high literacy helping to give meaning to uncertain results. ${ }^{33}$ However, misconceptions about results and inadequate information caused confusion and uncertainty about the future for some participants, ${ }^{25,26,32}$ as well as difficulty making decisions. ${ }^{28}$ Bradbury and colleagues $^{28}$ found that making a less informed choice (which was defined as inadequate knowledge and a decision discordant with attitudes toward testing) about genomic testing was associated with greater uncertainty prior to testing. While previous experience with uncertainty and disease led some participants to believe they could cope with the uncertainty related to genomic testing, ${ }^{26,33}$ Bradbury and colleagues $^{28}$ found that previous experience with genetic testing was not associated with participants' mean baseline uncertainty scores.

Health professionals' credentials, and communication approach and style when discussing genomic results, ${ }^{25,26,28,32,33}$ also influenced participants' uncertainty. Participants preferred to have a genetic counselor rather than a medical geneticist or oncologist explain their germline genomic results, ${ }^{33}$ and found that uncertainty was increased when health-care professionals provided too much information causing information overload, or used genetic/medical language, such as "not interpretable."

A trust in science influenced patients' experience of uncertainty about treatment decisions based on genomic results, with trusting participants feeling more certain about their targeted or standard treatment. ${ }^{32}$ These participants trusted the science, therefore any genomic result was reassuring and reduced uncertainty to some extent. ${ }^{32}$

\footnotetext{
"The tests showed that in any case, I had to be given docetaxel, which was the only possible option, and therefore I wasn't included in the trial protocol. So in the end I underwent the normal, well-known procedure, which is quite reassuring." 32
}

The influence of the type of genomic result and participant uncertainty was mixed. While the quantitative synthesis showed no difference in uncertainty levels between participants who received positive, negative, or uncertain genomic results, ${ }^{30,31}$ the qualitative synthesis found decreases in disease and risk uncertainty for participants who received negative or positive genomic results, but increased uncertainty about the future for participants receiving a VUS result. ${ }^{25,28,32,33}$ 
"It's just that I don't know...I know I've got something, but I don't know what and I don't know what it means." ${ }^{25}$

Some participants expressed tolerance for the uncertainty involved in receiving VUS results; ${ }^{33}$ others who received uncertain genomic results subsequently reported feeling more uncertain. $^{28,33}$ Participants' experience of uncertain results depended on whether they appraised the uncertainty attached to the result as a danger, an opportunity, or both. ${ }^{25,26}$ Those who appraised the uncertainty as a danger considered their VUS as a health threat (i.e., assumed high risk). Those who appraised uncertainty as an opportunity held hope for a positive outcome (i.e., not developing cancer). Some participants appraised the VUS as both an opportunity and a danger, seeing it as a health threat but not as serious a threat as a positive result.

\section{Outcomes of uncertainty}

Lumish and colleagues ${ }^{30}$ reported that participants receiving uncertain genomic results misinterpreted their VUS result as a negative result. Esteban and colleagues ${ }^{31}$ reported that participants who receive uncertain genomic results have similar psychological reactions as participants who receive negative genomic results, including lower levels of distress and higher levels of positive experience compared with those who receive a positive genomic result.

Participants who received uncertain genomic results experienced a range of affective reactions to their results, including frustration, shock, regret, sadness, disappointment, and further uncertainty about the future. ${ }^{25,26,32}$ Some participants acknowledged that once you experience uncertainty, you cannot go back to being oblivious. ${ }^{33}$

"I can't un-know that information." 33

Some participants did also experience a sense of relief that something (however uncertain) was detected. ${ }^{25}$

"Yeah, it was a relief in yeah okay I do have something that it's not my fault I got cancer and the other part of me was like this doesn't mean anything." 25

Uncertainty also influenced participants' decisions regarding communicating genomic results to relatives. Participants assessed whether the information would be more harmful than useful to their relatives, and judged uncertain results to be more potentially harmful and difficult to communicate, generating hesitancy to share such results with relatives. ${ }^{33}$

"...if it was going to do more harm than good by telling them certain results, there is a slight chance I would not tell them."33

\section{Coping with uncertainty}

Participants were mostly individuals with a personal and/or family history of cancer. Having experienced cancer-related uncertainty, they were willing to risk more uncertainty for the chance of some certainty about their cancer, and/or future risk. Previous experience of coping with uncertainty gave participants a sense of self-efficacy to be able to cope with more uncertainty if that was the outcome of genomic testing. ${ }^{26,33}$

Prior to receiving genomic results, participants felt that they would respond to uncertain results by seeking more information, or by remaining optimistic about a positive future outcome. ${ }^{25,33}$ Subsequently many participants did cope with their uncertainty by seeking more information and by remaining optimistic that their VUS would be reclassified in the future. Researching what was known about their uncertain result and what clinical trials were available based on that result helped participants cope with the uncertainty of their genomic results. ${ }^{25,33}$

"I didn't want to get a second opinion because it was a big deal to get a genetic work-up, and I trusted the geneticist. I went to the Internet, though, and started doing some research on my own." 25

Some participants felt that their faith or positive attitude helped them to be resilient enough to cope with uncertainty, through believing that the positive outcome would occur. ${ }^{33}$ Some participants appraised uncertain genomic results as an opportunity, believing that advances in science would provide more certain answers in the future. ${ }^{25}$

"I feel that there is a seed of hope that I could be completely normal. Some information could come out in five years, ten years, twenty years, that could make me interpret these results differently." 25

Additionally, participants coped with uncertainty by complying with recommended screening, sharing risk information with relatives, or by forming a plan of action based on high risk cancer assumptions. ${ }^{25,26,33}$ Participants who appraised an uncertain genomic result as a danger selfidentified as high risk or as having a positive result and acted accordingly in terms of risk reduction strategies. ${ }^{25}$

\footnotetext{
"And getting my ovaries out, that was a hard decision but the thing is I don't want to die of something that doesn't do anything. I would rather just go ahead and get them out. I want to live. Definitely safe vs. sorry, absolutely." 25
}

Regardless of whether participants appraised the uncertainty as an opportunity or danger, coping strategies involved having a plan. Participants believed that they could intervene with their behavior to reduce their risk with surveillance and management plans. $^{25,26}$

"You know the thing is you need to get a plan in place, like get a surveillance plan in place, know what you're going to be doing and that gives me more comfort. To think every 
year, okay I'm going to have a colonoscopy and you know I'm going to let the doctors know about it...."25

Some participants coped with uncertainty by sharing their genomic information with relatives, stating that this gave them a sense of protecting their relatives with risk information. ${ }^{25}$

\section{DISCUSSION}

This systematic review provides a synthesis of the existing research on the patient experience of uncertainty related to cancer genomic testing. Overall, our findings highlight the multiplicity of coexisting uncertainties involved in genomic testing for patients, supporting what has been found in the literature on the experience of uncertainty in patients who are pursuing cancer genetic testing. ${ }^{12,15,16,38-44}$

To ensure that the findings of this systematic review were grounded in the data, we focused only on the data from the papers included in the review during the analysis, rather than using theories or frameworks to guide analysis. It is important however to discuss these results in the context of the wellknown theories and frameworks of uncertainty. Our results align with Han's taxonomy of sequencing uncertainty. ${ }^{6}$ For instance the source of patient's uncertainty may relate to the imprecise nature and usefulness of genomic results, or the complexity with which results are delivered. Scientific uncertainty felt by some patients may be a result of low genomic literacy, while personal uncertainty may occur as a consequence of not knowing how they may react psychologically to their genomic results.

Our results suggest that how a patient appraises uncertainty, as either a danger, opportunity, or both, has implications initially for whether patients will decide to pursue cancer genomic testing; and subsequently for the impact of uncertainty for patients, as well as the coping strategies engaged to deal with their uncertainty. This appraisal of uncertainty as opportunity or danger is supported by Mishel's ${ }^{7}$ uncertainty in illness theory, which states that the experience of uncertainty is neutral until the implications of uncertainty are determined by the patient. When uncertainty is appraised as a danger, strategies to reduce the uncertainty, such as information seeking, are engaged. When uncertainty is seen as an opportunity, strategies to maintain uncertainty or reframe the uncertainty into a positive outcome, such as focusing on the positive aspects of unknowns, are implemented. ${ }^{7,45}$ Similarly, this systematic review found that those who viewed their uncertain cancer genomic results as an opportunity remained hopeful that research would evolve and provide a more certain result in the future; while those who appraised their uncertain cancer genomic result as a danger acted to reduce their uncertainty by implementing risk management action plans based on a high risk or positive result.

Appraisal involves evaluation of uncertainty, which is influenced by personality, previous experience, knowledge, contextual cues, and beliefs. ${ }^{7,45}$ Simpkin et al. ${ }^{46}$ and Biesecker et al. ${ }^{45,47}$ have discussed the importance of more effectively communicating the practical and scientific uncertainties of genomics with patients to better manage patient expectations and subsequent uncertainties postresults. This systematic review also found that health-care professionals can help reduce patient uncertainty by communicating in lay language.

This review highlighted that there is currently limited published research on the patient experience of uncertainty when pursuing cancer genomic testing. To more comprehensively assess the limited research available, two hypothetical studies were included in this systematic review. We recognize the limitations of hypothetical studies, and that findings may not be generalizable to real clinical situations. However, participants' responses regarding the types of uncertainty they expected to experience and the factors influencing that uncertainty are likely representative of their actual opinions and attitudes. ${ }^{24,26}$ It should also be noted that patient experiences of uncertainty reported in the hypothetical studies were similar to those reported in studies reporting on real-life use of testing.

Due to the scarcity of literature on any one type of genomic test, we included studies on a variety of genomic tests in a cancer context. There did not appear to be differences in the experience of uncertainty across the test types, for example exome sequencing compared with SNP testing. The only difference that emerged was as a result of the purpose of the genomic testing: providing risk information versus treatment indications, where genomic results decreased decisional uncertainty for patients in the cancer treatment context, compared with no change in uncertainty for patients receiving cancer risk genomic results. ${ }^{27-29,31}$

Much of what we know about the psychosocial issues of cancer patients related to genetic or genomic testing is from an overrepresented female and breast cancer perspective. ${ }^{48}$ Similarly, the majority of research investigating patient uncertainty in relation to cancer genomic has focused on females with a personal or family history of breast cancer, highlighting a bias in the literature and a need for future research to include a more diverse range of cancer patients. Future research should include patients with rarer cancers, or cancers with fewer treatment options, to understand more clearly how cancer genomic testing compounds or mitigates disease uncertainty.

Mishel's reconceptualized uncertainty in illness theory ${ }^{8}$ suggests that uncertainty evolves over time, stating that the longer a patient lives with a chronic illness and continual uncertainty, the more positively they appraise their uncertainty. For example, an initial appraisal of uncertainty as danger may evolve into appraisal of uncertainty as opportunity over time. This is supported in the cancer genetic literature by Halbert and colleagues ${ }^{49}$ finding that women experiencing uncertainty had received their $B R C A$ results more recently than women who were not experiencing uncertainty. Of the few studies included in our systematic review that did follow patients over time, most focused on the receipt of uncertain genomic results only ${ }^{27-29}$ and only one 
reported on psychosocial outcomes of uncertainty. ${ }^{31}$ Further longitudinal research focusing on uncertainty is needed to understand the how the experience of uncertainty changes over time, and the impact of that uncertainty.

\section{Conclusion}

This systematic review highlights the need for more mixed methods longitudinal research to understand the experience of patient uncertainty across the genomic testing process (e.g., waiting for and receiving results, and making treatment/risk management decisions), as well as the long-term psychosocial impacts of uncertainty for a broad range of cancer patients. The evidence highlights that while patients approach genomic testing as a disease uncertainty reduction strategy, genomic testing can also raise its own uncertainties for patients. It appears that appraisal of uncertainty as a danger, an opportunity, or both, is important in how the patient experiences uncertainty, as well the impact that uncertainty will have for them and the coping strategies that they utilize.

\section{SUPPLEMENTARY INFORMATION}

The online version of this article (https://doi.org/10.1038/s41436020-0829-y) contains supplementary material, which is available to authorized users.

\section{ACKNOWLEDGEMENTS}

The PiGeOn Project is funded by a National Health and Medical Research Council (NHMRC) of Australia Project Grant (ID 1124749). N.B. is funded by a Sydney Catalyst Postgraduate Research Scholarship. P.B. is funded by a National Health and Medical Research Council (NHMRC) Senior Principal Research Fellowship. MB is funded by a Post-Doctoral Research Fellowship from the Cancer Institute of NSW. These grants are competitive and involve peer review.

\section{DISCLOSURE}

The authors declare no conflicts of interest.

Publisher's note Springer Nature remains neutral with regard to jurisdictional claims in published maps and institutional affiliations.

\section{REFERENCES}

1. Bray F, Ferlay J, Soerjomataram I, Siegel RL, Torre LA, Jemal A. Global cancer statistics 2018: GLOBOCAN estimates of incidence and mortality worldwide for 36 cancers in 185 countries. CA Cancer J Clin. 2018;68:394-424.

2. National Cancer Institute. The genetics of cancer. 2017. https://www cancer.gov/about-cancer/causes-prevention/genetics. Accessed February 2018.

3. Lynce F, Isaacs C. How far do we go with genetic evaluation? Gene panel, and tumor testing. Am Soc Clin Oncol Educ Book. 2016;36: e72-e78.

4. Ormond KE, Wheeler MT, Hudgins $L$, et al. Challenges in the clinical application of whole-genome sequencing. Lancet. 2010;375: 1749-1751.

5. Robson M, Bradbury A, Arun B, et al. American Society of Clinical Oncology policy statement update: genetic and genomic testing for cancer susceptibility. J Clin Oncol. 2015;33:3660.
6. Han P, Umstead K, Bernhardt B, et al. A taxonomy of medical uncertainties in clinical genome sequencing. Genet Med. 2017;19:918-925.

7. Mishel M. Uncertainty in illness. J Nurs Scholarsh. 1988;20:225-232.

8. Mishel M. Reconceptualization of the uncertainty in illness theory. J Nurs Scholarsh. 1990:22:256-262.

9. Han P, Klein W, Arora N. Varieties of uncertainty in health care: a conceptual taxonomy. Med Decis Making. 2011;31:828-838.

10. Eisenberg S, Kurita K, Taylor-Ford M, Agus D, Gross M, Meyerowitz B. Intolerance of uncertainty, cognitive complaints, and cancer-related distress in prostate cancer survivors. Psychooncology. 2015;24:228-235.

11. Kurita K, Garon E, Stanton A, Meyerowitz B. Uncertainty and psychological adjustment in patients with lung cancer. Psychooncology. 2013;22:1396-1401.

12. Baty B, Dudley W, Musters A, Kinney A. Uncertianty in BRCA1 cancer susceptibility testing. Am J Med Genet C. 2006;142C:241-250.

13. Khan C, Moore E, Leos C, Rini C. Patient hopes for diagnotic genomic sequencing: roles of uncertianty and social status. Eur J Hum Genet. 2016;24:803-808.

14. Vos J, Otten W, van Asperen C, Jansen A, Menko F, Tibben A. The counsellees' view of an unclassified variant in BRCA1/2: recall, interpretation, and impact on life. Psychooncology. 2008;17:822-830.

15. Dean M. "It's not if I get cancer, it's when I get cancer": BRCA-positive patients' (un)certain health experiences regarding hereditary breast and ovarian cancer risk. Soc Sci Med. 2016;163:21-27.

16. Dean M, Scherr CL, Clements M, Koruo R, Martinez J, Ross A. "When information is not enough": a model for understanding BRCA-positive previvors' information needs regarding hereditary breast and ovarian cancer risk. Patient Educ Couns. 2017;100:1738-1743.

17. Kerr A, Swallow J, Chekar CK, Cunningham-Burley S. Genomic research and the cancer clinic: uncertainty and expectations in professional accounts. New Genet Soc. 2019;38:222-239.

18. Moher D, Liberati A, Tetzlaff J, Altman DG. Preferred reporting items for systematic reviews and meta-analyses: the PRISMA statement. BMJ. 2009;339:b2535.

19. Tong A, Flemming K, Mclnnes E, Oliver S, Craig J. Enhancing transparency in reporting the synthesis of qualitative research: ENTREQ. BMC Med Res Methodol. 2012;12:181

20. Green ED, Guyer MS, National Human Genome Research I, et al. Charting a course for genomic medicine from base pairs to bedside. Nature. 2011;470:204.

21. Aromataris E, Munn Z Joanna Briggs Institute reviewer's manual. Joanna Briggs Institute; 2017. https://reviewersmanual.joannabriggs.org/.

22. Thomas J, Harden A, Oakley A, et al. Integrating qualitative research with trials in systematic reviews. BMJ. 2004;328:1010-1012.

23. Thomas J, Harden A. Methods for the thematic synthesis of qualitative research in systematic reviews. BMC Med Res Methodol. 2008;8:45.

24. Braithwaite D, Sutton S, Steggles N. Intention to participate in predictive genetic testing for hereditary cancer: the role of attitude toward uncertainty. Psychol Health. 2002;17:761-772.

25. Solomon I, Harrington E, Hooker G, et al. Lynch syndrome limbo: patient understanding of variants of uncertain significance. J Genet Couns. 2017:26:866-877

26. Leventhal KG, Tuong $W$, Peshkin BN, et al. "Is it really worth it to get tested?": primary care patients' impressions of predictive SNP testing for colon cancer. J Genet Couns. 2013:22:138-151.

27. Holt S, Bertelli G, Humphreys I, et al. A decision impact, decision conflict and economic assessment of routine Oncotype DX testing of 146 women with node-negative or pNImi, ER-positive breast cancer in the U.K. Br J Cancer. 2013:108:2250-2258.

28. Bradbury AR, Patrick-Miller L, Egleston BL, et al. Patient feedback and early outcome data with a novel tiered-binned model for multiplex breast cancer susceptibility testing. Genet Med. 2015;18:25.

29. Levine MN, Julian JA, Bedard PL, et al. Prospective evaluation of the 21 gene recurrence score assay for breast cancer decision-making in Ontario. J Clin Oncol. 2016:34:1065-1071.

30. Lumish HS, Steinfeld $\mathrm{H}$, Koval $\mathrm{C}$, et al. Impact of panel gene testing for hereditary breast and ovarian cancer on patients. J Genet Couns. 2017:26:1116-1129.

31. Esteban I, Vilaro M, Adrover $\mathrm{E}$, et al. Psychological impact of multigene cancer panel testing in patients with a clinical suspicion of hereditary cancer across Spain. Psychooncology. 2018;27:1530-1537.

32. Pellegrini I, Rapti M, Extra J-M, et al. Tailored chemotherapy based on tumour gene expression analysis: breast cancer patients' misinterpretations and positive attitudes. Eur J Cancer Care. 2012;21:242-250. 
33. Hitch K, Joseph G, Guiltinan J, Kianmahd J, Youngblom J, Blanco A Lynch syndrome patients' views of and preferences for return of results following whole exome sequencing. J Genet Couns. 2014;23:539-551.

34. Cella D, Hughes $C$, Peterman A, et al. A brief assessment of concerns associated with genetic testing for cancer: The multidimensional impact of cancer risk assessment (MICRA) questionnaire. Health Psychol. 2002;21:564-572.

35. O'Connor AM. Validation of a decisional conflict scale. Med Decis Making. 1995;15:25-30.

36. O'Connor AM. User manual- decisional conflict scale (10 item question format). 1993. https://decisionaid.ohri.ca/docs/develop/User_Manuals/ UM_Decisional_Conflict.pdf.

37. Slavin TP, Neuhausen SL, Nehoray B, et al. The spectrum of genetic variants in hereditary pancreatic cancer includes Fanconi anemia genes. Fam Cancer. 2018;17:235-245.

38. Claes E, Evers-Kiebooms G, Boogaerts A, Decruyenaere M, Denayer L, Legius $E$. Diagnostic genetic testing for hereditary breast and ovarian cancer in cancer patients: women's looking back on the pretest period and a psychological evaluation. Genet Test. 2004;8:13-21.

39. Claes E, Denayer L, Evers-Kiebooms G, Boogaerts A, Legius E. Predictive testing for hereditary non-polyposis colorectal cancer: motivation, illness representations and short-term psychological impact. Patient Educ Couns. 2004;55:265-274.

40. Hallowell N, Foster C, Ardern-Jones A, Eeles R, Murday V, Watson M. Genetic testing for women previously diagnosed with breast/ovarian cancer: examining the impact of BRCA1 and BRCA2 mutation searching. Genet Test. 2002;6:79-87.

41. Lim J, Macluran M, Price M, Bennett B, Butow P. Short- and long-term impact of receiving genetic mutation results in women at increased risk for hereditary breast cancer. J Genet Couns. 2004;13:115-133.

42. d'Agincourt-Canning L. A gift or a yoke? Women's and men's responses to genetic risk information from BRCA1 and BRCA2 testing. Clin Genet. 2006;70:462-472.

43. van Roosmalen MS, Stalmeier PF, Verhoef LC, et al. Impact of BRCA1/2 testing and disclosure of a positive test result on women affected and unaffected with breast or ovarian cancer. Am J Med Genet A. 2004; 124a:346-355.
44. Cypowyj C, Eisinger F, Huiart L, Sobol H, Morin M, Julian-Reynier C. Subjective interpretation of inconclusive BRCA1/2 cancer genetic test results and transmission of information to the relatives. Psychooncology. 2009;18:209-215.

45. Biesecker $B B$, Klein $W$, Lewis $K L$, et al. How do research participants perceive "uncertainty" in genome sequencing? Genet Med. 2014;16:977.

46. Simpkin AL, Armstrong KA. Communicating uncertainty: a narrative review and framework for future research. J Gen Intern Med. 2019;34: 2586-2591.

47. Biesecker BB, Woolford SW, Klein WMP, et al. PUGS: a novel scale to assess perceptions of uncertainties in genome sequencing. Clin Genet. 2017:92:172-179.

48. Yanes T, Willis AM, Meiser B, Tucker KM, Best M. Psychosocial and behavioral outcomes of genomic testing in cancer: a systematic review. Eur J Hum Genet. 2019;27:28-35.

49. Halbert $\mathrm{CH}$, Stopfer JE, MCDonald J, et al. Long-term reactions to genetic testing for BRCA1 and BRCA2 mutations: does time heal women's concerns? J Clin Oncol. 2011;29:4302-4306.

\section{(i) (2)(2)}

Open Access This article is licensed under a Creative Commons Attribution-NonCommercial-ShareAlike 4.0 International License, which permits any non-commercial use, sharing, adaptation, distribution and reproduction in any medium or format, as long as you give appropriate credit to the original author(s) and the source, provide a link to the Creative Commons license, and indicate if changes were made. If you remix, transform, or build upon this article or a part thereof, you must distribute your contributions under the same license as the original. The images or other third party material in this article are included in the article's Creative Commons license, unless indicated otherwise in a credit line to the material. If material is not included in the article's Creative Commons license and your intended use is not permitted by statutory regulation or exceeds the permitted use, you will need to obtain permission directly from the copyright holder. To view a copy of this license, visit http://creativecommons.org/licenses/by-nc-sa/4.0/.

() The Author(s) 2020 